\title{
COMPARISON OF THREE APPROACHES ON DSP BASED DIGITAL CONTROLLED 2-WHEELER FORWARD CONVERTER
}

\author{
C.K. Lau \\ M.H. Pong \\ Department of Electrical and Electronic Engineering \\ The University of Hong Kong \\ Pokfulam Road, Hong Kong
}

\begin{abstract}
This paper presents the design of digital controls of PWM DC-DC converters on a digital signal processor (DSP) based platform. Three digital control approaches are being investigated: Discretisation of analog control, Digital proportional-integral-derivative (PID) control, and Deadbeat control. Simulation results of the three types of control are shown. Experimental results of a $25 \mathrm{~W} 2$-wheeler forward converter prototype are presented to verify the design.
\end{abstract}

\section{INTRODUCTION}

The recent advance in microprocessor technology has given better possibilities and advantages for using digital means in control of PWM DC-DC converter in replacement of the traditional analog control using op-amp based compensators. For digital control of PWM DC-DC converter, processing speed of the DSP is of vital importance, comparable performance to analog controlled converter can only be obtained with new high speed processors. The compensation network affects the stability and the frequency response of the power converter. With digital control, the design is highly flexible and is less dependent on the accuracy and stability of active and passive components used in analog compensators. Digital control permits one to realise sophisticated control laws which are difficult, and sometimes impossible, to realise by analog means. The simulation and experimental results of a $25 \mathrm{~W}$ $20 \mathrm{kHz}$ 2-wheeler forward converter prototype built on a TMS320C30 DSP platform verified the three approaches.

\section{CONTINUOUS AND DISCRETISED SYSTEM}

The 2-wheeler forward topology with a second order output filter is chosen as an example of the applications of the digital control schemes.

The control loop of the isolated 2-wheeler forward converter is divided into three main stages: power circuit, pulse width modulator, and error amplifier compensation network as shown on Fig. 1. The schematic and equivalent circuit diagrams of the power circuit are shown in Fig. 2 and 3 respectively. The block diagram of the DSP controlled forward converter is shown in Fig. 4.
Closed loop transfer function of the system is:

$C L T F=\frac{K_{p} K(s) G(s)}{1+K_{p} K(s) G(s)}$

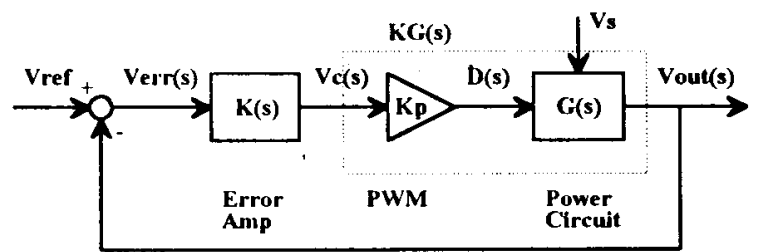

Fig. 1. Transfer function block diagram of the power converter

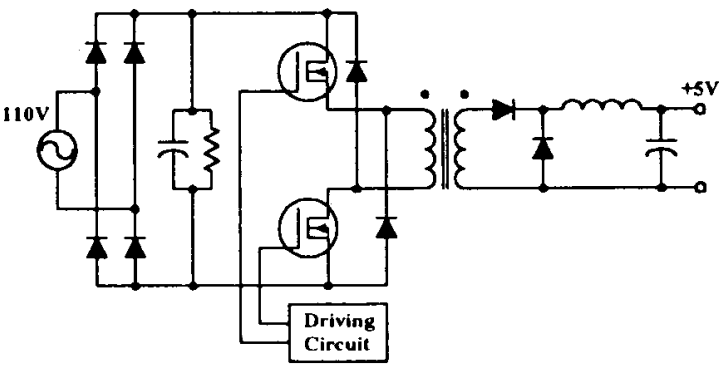

Fig. 2. Schematic diagram of the 2-wheeler forward converter

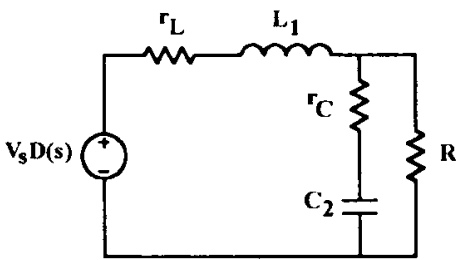

Fig. 3. Equivalent circuit of the power circuit

The transfer function of the equivalent power circuit in Fig. (2) is:

$K G(s)=\begin{aligned} & \frac{V_{s} \bullet\left(s R C_{2} r_{C}+R\right) \bullet D}{\left\{s^{2} C_{2} L_{1}\left(R+r_{C}\right)+s\left[R C_{2} r_{L}+C_{2} r_{L}\left(R+r_{C}\right)+L_{1}\right]\right.} \\ & \left.+r_{L}+R\right\} \bullet V_{C}\end{aligned}$ 


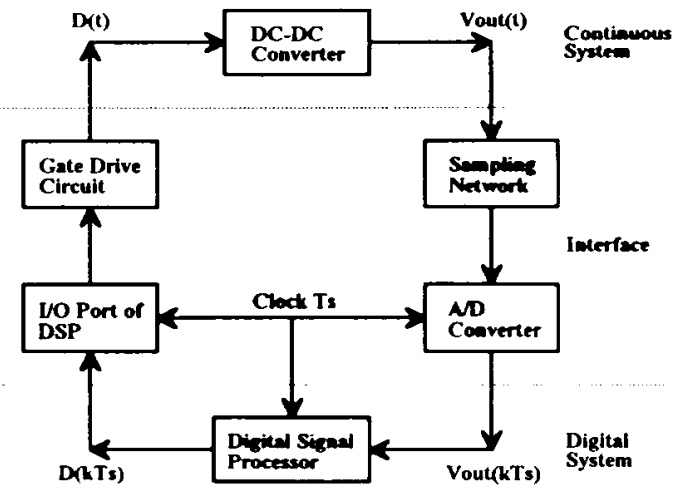

Fig. 4. Block diagram of DSP controlled forward converter

The input and output of DSP are related by a linear difference equation with constant coefficients:

$D\left(k T_{s}\right)+a_{1} D\left((k-1) T_{s}\right)+a_{2} D\left((k-2) T_{s}\right)+\ldots+a_{k} D(0)$

$=b_{0} V_{e r r}\left(k T_{s}\right)+b_{1} V_{e r r}\left((k-1) T_{s}\right)+b_{2} V_{e r r}\left((k-2) T_{s}\right)$

$+\ldots+b_{k} V_{e r r}(0)$

where $V_{\text {err }}\left(k T_{s}\right)=V_{\text {out }}\left(k T_{s}\right)-V_{\text {ref }}$

Taking z-transform of (3) and using backward shift property,

$K(z)=\frac{D(z)}{V_{\text {err }}(z)}=\frac{b_{0} z^{k}+b_{1} z^{k-1}+\ldots+b_{k}}{z^{k}+a_{1} z^{k-1}+a_{2} z^{k-2}+\ldots+a_{k}}$

where $K(z)$ is the Pulse Transfer Function (PTF) of the DSP controller.

After the continuous system is discretised by z-transform, the $\mathrm{ZOH}$ equivalent of $G(s)$ is:

$G_{0}(z)=\frac{\left(1-z^{-1}\right)}{z} Z\left[\frac{G(s)}{s}\right]=K\left[\frac{z^{2}+N_{1} z+N_{2}}{z^{2}+D_{1} z+D_{2}}\right]$

where

$K=\frac{V_{s} R r_{c}}{L_{1}\left(R+r_{C}\right)}$,

$N_{1}=-\left\{\frac{b}{\omega_{o}^{2}+a^{2}}\left[2 e^{-a T_{s}} \cos \left(\omega_{0} T_{s}\right)-\left(e^{-\Delta T_{s}} \cos \left(\omega_{0} T_{s}\right)+1\right)\right]\right.$

$\left.\left.-\frac{1}{\omega_{o}}\left(1-\frac{a b}{\omega_{o}^{2}+a^{2}}\right) e^{-a T_{s}} \sin \left(\omega_{o} T_{s}\right)\right]\right\}$.

$D_{1}=-2 e^{-a T_{s}} \cos \left(\omega_{o} T_{s}\right)$ and $D_{2}=e^{-2 a T_{s}}$.

$a=\frac{2 R C_{2} r_{L}+C_{2} r_{c} r_{L}+L_{1}}{2 C_{2} L_{1}\left(R+r_{c}\right)}, \quad b=\frac{1}{C_{2} r_{c}}$,

$\omega_{o}=\sqrt{\frac{r_{L}+R}{C_{2} L_{1}\left(R+r_{C}\right)}-a^{2}}$

\section{IIL. DIGITAL CONTROL ALGORITHMS}

(l) Discretisation of Analog Compensation Network

Analog compensation network can be discretised by bilinear transformation. The resultant PTF of the discretised analog controller is

$$
\begin{aligned}
K(z) & =K\left(\frac{2}{T_{S}} \frac{1-z^{-1}}{1+z^{-1}}\right) \\
& =\frac{\left(2 T_{S}+s_{z 1}\right) z^{2}+\left(2 s_{z 1} T_{S}^{2}\right) z+\left(s_{z 1} T_{S}^{2}-2 T_{S}^{2}\right)}{\left(4+2 s_{p 1} T_{S}\right) z^{2}-8 z+\left(4-2 s_{p 1} T_{S}\right)}
\end{aligned}
$$

By inverse $z$-transform, $K(z)$ is transformed into a difference equation which can be implemented by the DSP. The design is further optimised through simulation and the final difference equation of the discretised analog controller is

$$
\begin{aligned}
D(k)= & 0.0884 V_{e r r}(k)+0.0026 V_{\text {err }}(k-1)-0.0858 V_{\text {err }}(k) \\
& +0.6131 D(k-1)+0.3869 D(k-2)
\end{aligned}
$$

\section{(2) Digital PID control}

For analog PID controller, the transfer function is:

$K(s)=K_{p}+K, \frac{1}{s}+K_{d} s$

where $K_{p}$ is the proportional gain, $K_{i}$ is the integral gain, $K_{d}$ is the derivative gain

Similar to s-domain, the integrator and differentiator can be represented by PTF in z-domain as

$K(z)=K_{p}+K_{i} \frac{T_{S}(z+1)}{2(z-1)}+K_{d} \frac{z-1}{T_{S} z}$

The design is further optimised through simulation and the final difference equation of the digital PID controller is:

$$
\begin{aligned}
D(k)= & 0.7824 V_{\text {err }}(k)-1.1368 V_{e r r}(k-1) \\
& +0.575 V_{e r r}(k-2)+D(k-1)
\end{aligned}
$$

\section{(3) Deadbeat control}

Deadbeat control is also called Finite Settling Time Control which forces the output of the system to the expected value after a finite number of sampling intervals.

The difference equation is:

$$
\begin{aligned}
& V_{-r r}(k+2)+\left(D_{2}-D_{1}^{2}\right) \operatorname{Verr}(k)-D_{1} D_{2} V_{\text {err }}(k-2) \\
& =K N_{1} D(k)+K\left(N_{2}-N_{1} D_{1}\right) D(k-1)-K N_{2} D_{1} D(k-2)
\end{aligned}
$$

Putting $V_{\text {err }}(k+2)=0$ into (11) since the digital controller has one sampling delay, 


$$
\begin{aligned}
D(k)= & \frac{D_{2}-D_{1}^{2}}{K N_{1}} V_{e r r}(k)-\frac{D_{1} D_{2}}{K N_{1}} V_{e r r}(k-1) \\
& +\frac{N_{1} D_{1}-N_{2}}{N_{1}} D(k-1)+\frac{N_{2} D_{1}}{N_{1}} D(k-2)
\end{aligned}
$$

After optimisation through simulation, the resultant difference equation is found to be:

$$
\begin{aligned}
D(k)= & -2.069 V_{e r r}(k)+1.2222 V_{e r r}(k-1) \\
& -1.4398 D(k-1)+0.4094 D(k-2)
\end{aligned}
$$

\section{SIMULATION RESULTS}

Using the equivalent power circuit model described above in Fig. 3, frequency response simulations of the three digital controllers are done to analyse the stability of the control loop of the system.

The simulated frequency response of the discretised analog controlled, digital PID controlled and deadbeat controlled are shown in Fig. 5.
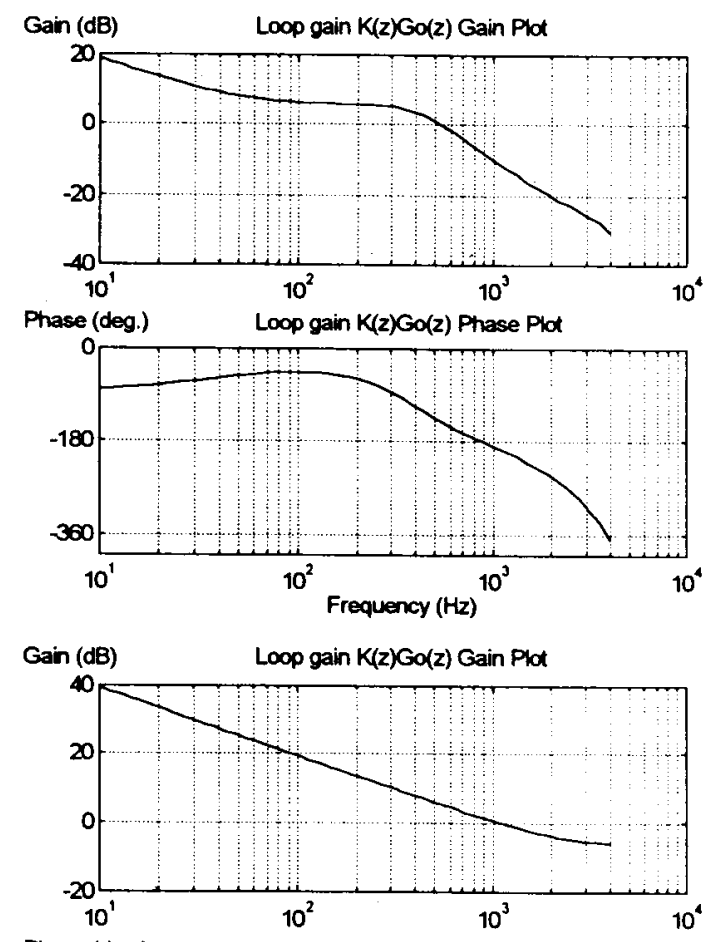

Phase (deg.) Loop gain $\mathrm{K}(\mathrm{z}) \mathrm{Go}(\mathrm{z})$ Phase Plot

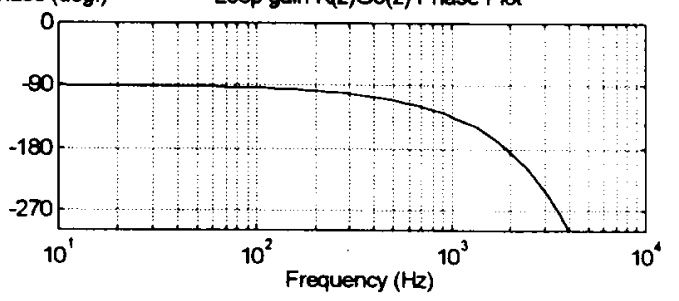

(a)

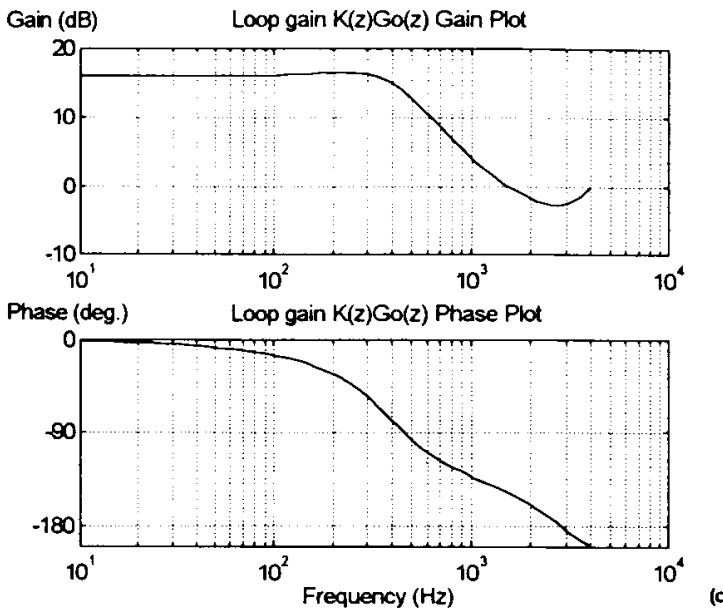

Fig. 5. Simulated frequency response of the (a) discretised analog controlled, (b) digital PID controlled and (c) deadbeat controlled power controllers

$V_{s}=17.4 \mathrm{~V}, r_{L}=0.2 \Omega, r_{C}=0.08 \Omega, L_{1}=180 \mu \mathrm{H}, C=1000 \mu \mathrm{F}$,

$R=1 \Omega$ and assuming continuous current

To compare the performance and stability of the controllers, the bandwidth, phase margin and the gain at $10 \mathrm{~Hz}$ are plotted as Fig. 6.
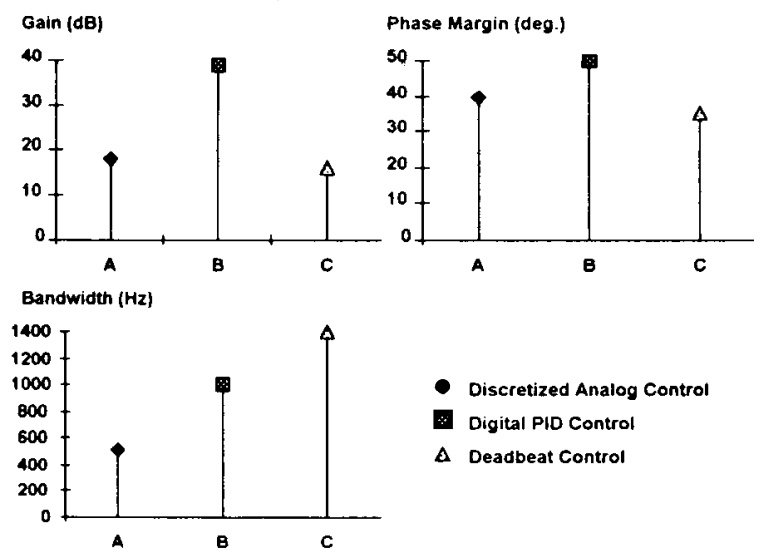

- Discretized Analog Control

Digital PID Control

A Deadbeat Control

Fig. 6. Comparisons of simulation results of the three digital controllers

\section{EXPERIMENTAL RESULTS}

\section{(1) Frequency Response}

A $25 \mathrm{~W} 20 \mathrm{kHz} 2$-wheeler forward converter is built to verify the control schemes proposed. The Texas Instrument TMS320C30 DSP running at 33.3 million floating point operations per second is used to implement the digital control algorithms.

Gain-phase plots of the power convener are measured to analyse the stability of the system as shown in Fig. 7. 


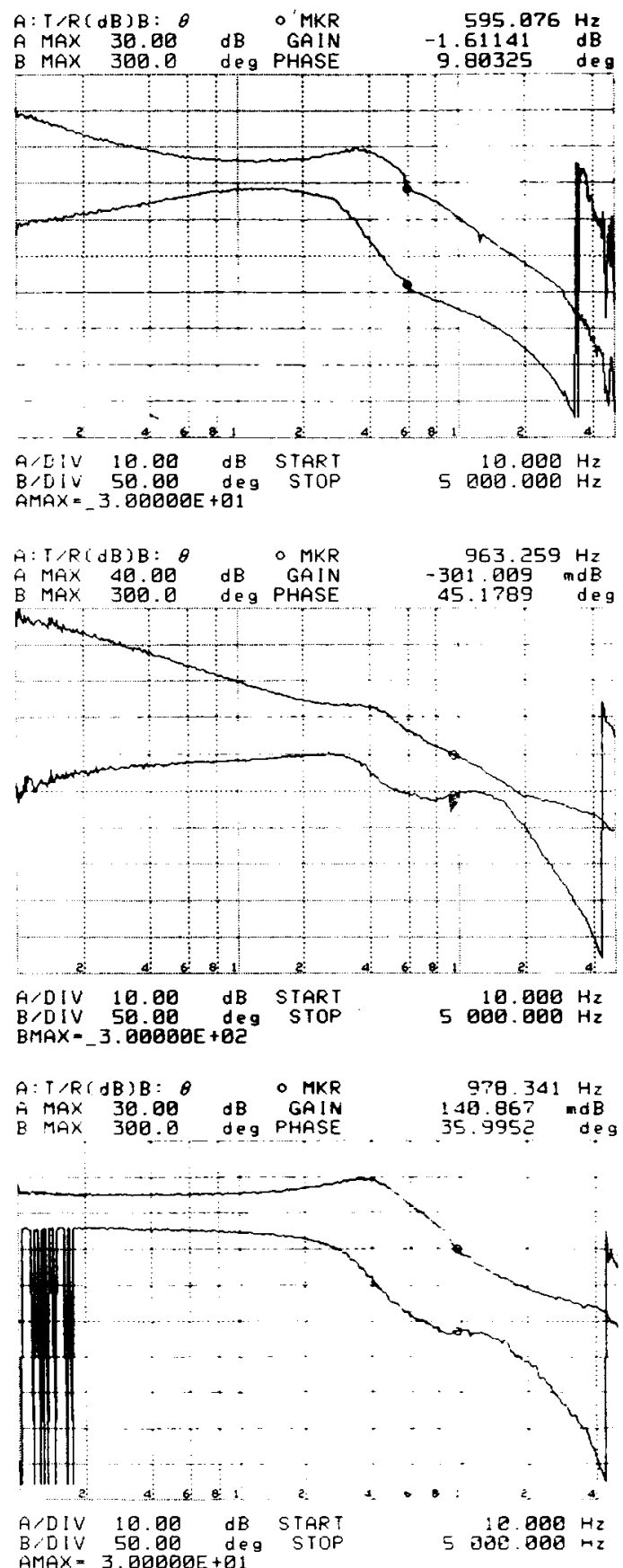

(a)

(b)

(c)

Fig. 7. Gain-phase plots of (a) discretised analog controlled, (b) digital PID controlled and (c) deadbeat controlled power controllers

To compare the performance and stability of the controllers, the bandwidth, phase margin and the gain at $10 \mathrm{~Hz}$ are plotted as Fig. 8 .
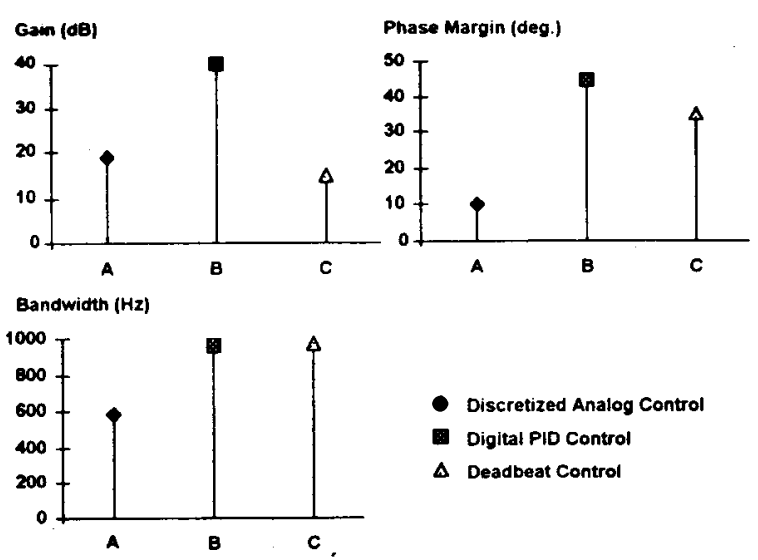

Discretized Analog Control

Digital PID Control

$\triangle$ Deadbeat Control

Fig. 8. Comparisons of experimental results of the three digital controllers

\section{(2) Transient Response}

Transient response is measured by switching the output load from 75 to 100 percent of its full load at a rate of 100 $\mathrm{Hz}$. Such a load change tests the stability and the response of the power converter. Fig. 9 shows the output of the power converter with different controllers under test.

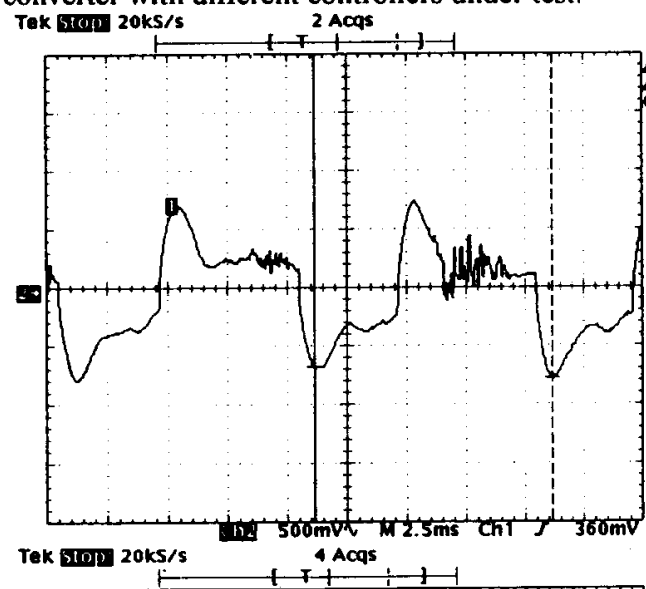

(a)

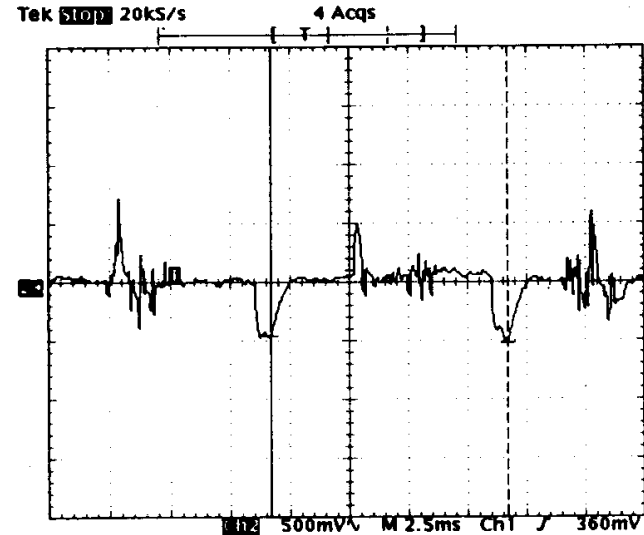

(b) 


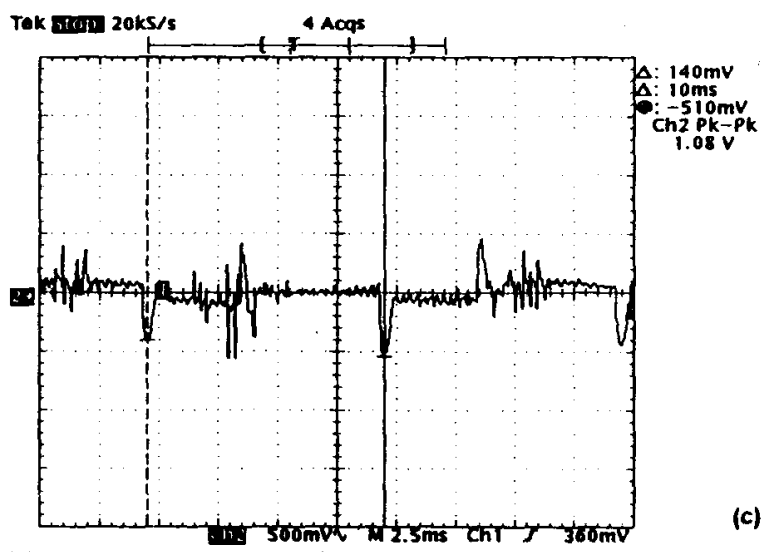

Fig. 9. Output voltage of (a) discretised analog controlled, (b) digital PID controlled, (c) deadbeat controlled power controllers

To compare the transient performance of the controllers, the magnitude of the transient and the maximum recovery time are measured as shown in Fig. 10.
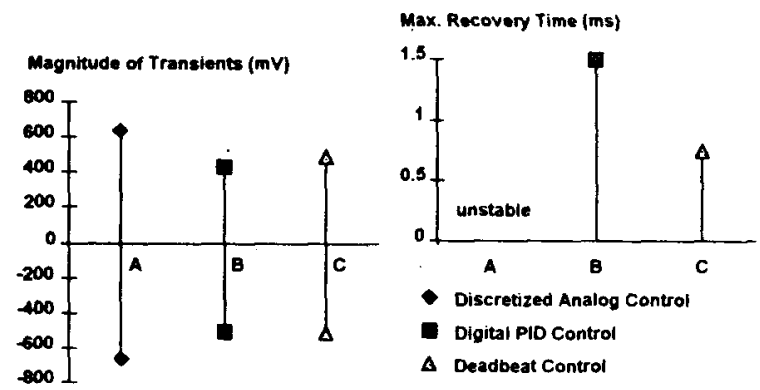

Fig. 10. Magnitude of the transient and the maximum recovery time of the power converters

The experimental results are found to follow very closely with the simulated results proving the validity of simulations being one of the advantages of digital control.

From the simulation results and experimental measurements obtained, it is shown that the stability of discretised analog controller is not as good as the others since it creates additional phase shift. The digital PID controller has the best performance among the three. The design of deadbeat controller is highly dependent on the accuracy of $\mathrm{G}(\mathrm{s})$ since the PTF is derived from the $\mathrm{ZOH}$ equivalent of $\mathrm{G}(\mathrm{s})$. For the deadbeat controller, the output error is forced to zero within two sampling periods. This implies a large magnitude of duty cycle. Since the range of duty cycle is limited, the requirement is not satisfied. Therefore the advantage of deadbeat controller is not obvious as shown in the transient test.

\section{CONCLUSION}

In this paper, a DSP controlled power converter is successfully designed with good response and stability using the three digital control approaches: discretised analog control, digital PID control and deadbeat control. Digital Control of PWM DC-DC converter is a highly feasible alternative of the traditional analog compensation due to its flexibility and accuracy. Digital control makes the realisation of sophisticated control laws possible. Experimental results, matching the simulation results have already proved the proposed control schemes.

\section{REFERENCE}

I. C.K. Tse, "Towards computer control for power supplies", Proceedings of First Hong Kong IEEE workshop on switch mode power supplies, December 1993, pp. 79-89.

2. Meng-Yueh Chang, Rong-Shyang, Ou, Ying-Yu Tzou: "DSP-based fuzzy control of bilateral IGBT PWM DC-to-AC and DC-to-DC converters for battery energy storage system", Proceedings of the IECON, November 1993, pp. 1117-1122

3. "TMS320C $3 x$ User's Guide", Texas Instruments.

4. "TMS320C3x Evaluation Module Technical Reference", Texas Instruments.

5. "TMS320C3x Peripheral Control Library", Texas Instruments. 